\title{
An Enhanced Solver for The Game of Amazons
}

\author{
Jiaxing Song \\ Department of Computing Science \\ University of Alberta \\ Edmonton, AB, Canada T6G 2E3 \\ Email: jiaxing2@ualberta.ca
}

\author{
Martin Müller \\ Department of Computing Science \\ University of Alberta \\ Edmonton, AB, Canada T6G 2E3 \\ Email: mmueller@ualberta.ca
}

\begin{abstract}
The game of Amazons is a modern board game with simple rules and nice mathematical properties. It has a high computational complexity. In 2001, the starting position on a $5 \times 5$ board was proven to be a first player win.

The enhanced Amazons solver presented here extends previous work in the following five ways: by building more powerful endgame databases, including a new type of databases for socalled blocker territories, by improving the rules for computing bounds on complex game positions, by local search to find tighter local bounds, by using ideas from combinatorial game theory to find wins earlier, and by using a df-pn based solver. Using the improved solver, the starting positions for Amazons on the $4 \times 5$, $5 \times 4,4 \times 6,5 \times 6$ and $4 \times 7$ boards were shown to be first player wins, while $6 \times 4$ is a second player win. The largest proof, for the $5 \times 6$ board, is presented in detail.
\end{abstract}

\section{INTRODUCTION}

\section{A. Games and Artificial Intelligence}

Since the beginnings of Artificial Intelligence (AI) in the 1950s, the relationship between games and AI research has been a reciprocal one. Games have finite state spaces, well defined rules and quantifiable goals. They therefore offer ideal testbeds for AI research. This leads to competitive opponents for human game players and also often offers interesting insights into the games.

The game of Amazons is a modern board game with simple rules yet high complexity [1]. With a typical branching factor in the hundreds of moves, Amazons is an ideal testbed for selective search algorithms. Since the board naturally breaks down into independent subgames as a game proceeds, the game also provides a nice test domain for applying combinatorial game theory.

\section{B. Outline}

This paper presents several techniques for solving small Amazons boards and applies them to determine the gametheoretic result on several small rectangular board sizes. It is organized as follows: Section I motivates AI research in games, introduces the game of Amazons, and summarizes related work and the contributions of this paper. Section II describes the methodology used for solving Amazons, including essential background on combinatorial game theory, and introduces tightened and relaxed bounds on the value of a game. Section III discusses the three types of local game databases built, with a focus on the new blocker territory databases. Section IV gives details on how bounds on the value of each type of area are computed and combined for solving a full-board Amazons position. Section V contains experimental results of the solver, including details of the $5 \times 6$ solution, results for test cases from harder board sizes, and statistics on the different types of local areas. The final Section VI discusses ideas for further improving the Amazons solver.

\section{Contributions}

The following are the main contributions of this paper:

- A strong evaluation function for computing correct bounds on the value of small full-board Amazons positions based on many types of local analysis.

- A df-pn based solver for Amazons which utilizes the evaluation components above and improves search efficiency, often by orders of magnitude.

- A technique for computing, storing and using databases of blocker territories.

- Two new static rules for improving the bounds on active local areas.

- A notation for expressing tightened and relaxed bounds on the value of a game.

- Techniques for exploiting knowledge about infinitesimals which often occur in Amazons, including an application of subzero thermography.

- An extensive empirical evaluation of the new solver.

- Solving the initial positions of Amazons on $4 \times 5,5 \times 4$, $4 \times 6,5 \times 6$ and $4 \times 7$ boards to be first player wins, and solving $6 \times 4$ to be a second player win.

\section{The Game of Amazons}

The game of Amazons was invented by Walter Zamkauskas of Argentina in 1988. It is a two-player board game played on a rectangular board, with standard size $10 \times 10^{1}$. Each player controls 4 amazons, or queens, which are placed on the edge of the board before a game starts. Two players Black and White move alternately until the player to move has no legal move and thereby loses the game. White usually plays first. The starting position of $5 \times 6$ Amazons is shown in Figure 1 .

A move in Amazons is comprised of two compulsory phases: queen move and arrow shot. First, a player moves

\footnotetext{
${ }^{1}$ Throughout the paper, board dimensions are presented in the format width $\times$ height
} 


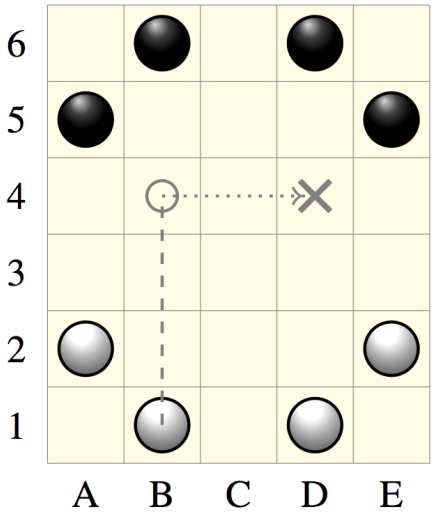

Fig. 1: $5 \times 6$ Amazons starting position and strong first move.

one queen $q$ of the player's own color from its origin square to a different destination square in a straight line either horizontally, vertically or diagonally, with the constraint that it may not cross or enter a square occupied by an amazon of either color or a burnt-off square. Next, $q$ has to shoot an arrow, which travels in the exact same way as a queen, from the square $q$ just moved to. The destination square of the arrow is burnt-off permanently from the board: no further queen moves or arrow shots can travel over or land on this square. Since exactly one empty square is burnt off in each move and at least one empty square is needed to make a move, an Amazons game is guaranteed to terminate in at most as many moves as there are empty squares. A strong opening move for White in $5 \times 6$ Amazons, shown in Figure 1 , is to move queen $B 1$ to $B 4$ and shoot to $D 4$, abbreviated as B1$\mathrm{B} 4 \times \mathrm{D} 4$. The gray circle in the figure marks the destination square for the queen, and the gray cross indicates the square to be burnt off.

\section{E. Related Work on Amazons}

Amazons has simple rules, but the computational complexity of all but the most trivial Amazons games is high due to its large branching factor. For example, the first player has 410 moves in the $5 \times 6$ starting position. Even the special case of deciding whether a queen can make a certain number of moves in one-player Amazons puzzles is NP-complete [2], and determining the winner of a generalized Amazons game is PSPACE-complete [3].

Since a board often breaks down into independent subgames as the game proceeds, Amazons has attracted the attention of researchers in combinatorial game theory. Berlekamp analyzed positions with one queen per player on $2 \times n$ boards and determined their thermographs [4]. Tegos built databases of combinatorial game values and defective territories, which cannot be completely filled, and used them for building a strong Amazons player [5]. Snatzke also computed the canonical forms of Amazons positions up to size $2 \times 11$ by exhaustive search [6], and both authors identified some interesting positions.

Kloetzer, Iida and Bouzy [7], [8] solve local endgames by several different minimax search methods, and compare algorithms for playing sums of such games. Okada et al. [9] $\mathrm{seem}^{2}$ to approximate a subgame by two integers.

\section{F. State of the Art for Solving Amazons}

Only a few results are known for analyzing or solving small Amazons boards. On boards with width or height less than four, there is no natural way to place the standard four amazons for each player. $1 \times n$ Amazons with one queen each is quite trivial. Let $n=b+w+s+2$, where $s$ is the number of "shared" empty points between the black and white amazon, and $b$ and $w$ the number of empty squares accessible only by black and white respectively. If $n=2$, then no queen can move and if $s=0$, the game is the number $b-w$ in terms of combinatorial game theory. Otherwise, each player can move to block the opponent and claim all the $s-1$ remaining shared points, and the game has the value $b-w \pm(s-1)$, with mean $b-w$ and temperature $s-1$.

Sums of $2 \times n$ positions with one queen per color per subgame were studied in detail by Berlekamp [4]. For the standard setup with four queens each, starting on the $(1,2)$ points in the corner, $4 \times 4$ Amazons "is a second player win, and can be solved easily by brute-force search [10]." $5 \times 5$ is a first player win [11].

\begin{tabular}{c||c|c|c|c} 
width \height & 4 & 5 & 6 & 7 \\
\hline \hline 4 & 2 & (1) & (1) & (1) \\
\hline 5 & (1) & 1 & (1) & $?$ \\
\hline 6 & (2) & $?$ & $?$ & $?$
\end{tabular}

TABLE I: Winners for Amazons on small boards. 1 means a first player win, 2 a second player win. New results are circled.

Table I summarizes the previously known and new results about solving Amazons on small boards. First player wins were found for the $4 \times 5,5 \times 4,4 \times 6,5 \times 6$ and $4 \times 7$ boards, while $6 \times 4$ turned out to be a second player win. The narrow and high versions seem much easier to prove as first player wins, since an effective first blocking move exists. $5 \times 4$ was much harder than $4 \times 5$, and while $4 \times 6$ is a fairly straightforward win by blocking, $6 \times 4$ is a second player win, and it took almost a full CPU day of computation to refute all 256 possible starting moves.

To the authors' knowledge, no results on other board sizes, with other numbers of queens, or with other starting locations of the queens have been published.

\section{BACKGROUND}

\section{A. Solving Amazons}

1) Simple Bounds: The deciding factor for analyzing an Amazons position is the difference in the number of moves that the players can make. If this number is zero, then the last player able to move wins. Simple integer bounds of a game $G$, as used in [11], limit the range of the number of moves Black can make more than White in $G$. Simple bounds of a

\footnotetext{
${ }^{2}$ The English summary of this Japanese paper is vague about this point.
} 
game are written in the form [lower, upper] where lower is a lower bound on this number, and upper is an upper bound. Given bounds $b$, the notation $l(b), u(b)$ is used to indicate these lower and upper bound respectively. For example, if the bounds of a game are $[-3,-1]$, then Black can make at least -3 and at most -1 more moves than White (i.e., White has at least 1 and at most 3 more moves than Black), therefore White wins.

The following rules [11] relate the winner of a game $G$ to its simple bounds $b=$ bounds $(G)$ :

- Black wins if $l(b)>0$, or if both $l(b)=0$ and it is White to move;

- White wins if $u(b)<0$, or if both $u(b)=0$ and it is Black to move;

- In other cases, bounds $b$ do not provide enough information to determine the winner of $G$.

These bounds and rules will be generalized in Section II-D.

2) Amazons Solver Architecture: Since neither arrows nor queens can pass through a burnt-off square, these squares establish a barrier between different parts of the board. As the game progresses and squares are burnt off, the board naturally splits into independent subgames (or areas). For example, in Figure 2, two such areas are delimited by solid lines.

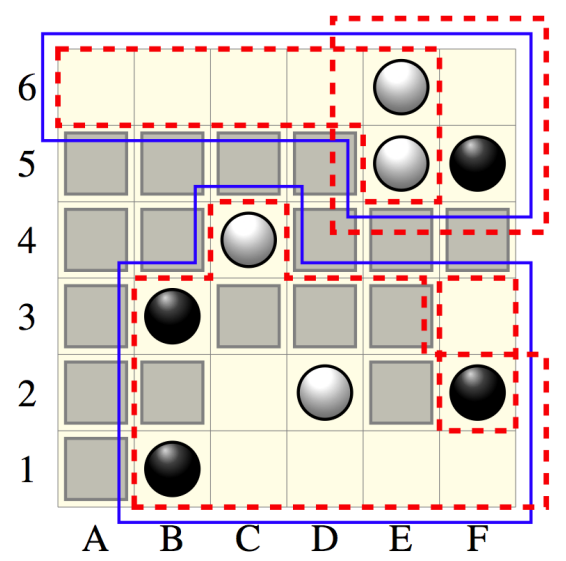

Fig. 2: Board partition.

Bounds on the values of independent areas can be computed individually and summed up into a single global bound, which can then be used to determine the winner of the whole board according to the rules above.

3) Board Partition: Intuitively, areas can be found by finding all the 8-connected components of empty squares and queens of both players on the board. This basic partition yields the two areas delimited by solid lines in Figure 2.

An improved partition can be achieved by using queens of one color to block the opponent [11]. In the basic partition, if part of an area is inaccessible to the opponent given that the player does not move, then this part of the board along with the blocking queens (called blockers) constitutes a new area, called a blocker territory. The non-blocker queens are called normal queens. In the example in Figure 2, the improved partitions are delimited by dashed lines. The White queens E5 and E6 and the Black queen $F 2$ are blockers. An improved partition splits an area from the basic partition into multiple smaller areas by using blockers. A blocker belongs to all the areas it separates, to illustrate the fact that it can potentially move in any of them.

4) Solver Overview: Algorithm 1 illustrates the abstract main loop of the solver. Function NextPosition gets the next position to be evaluated depending on the search algorithm, such as a most promising node in df-pn. The position is partitioned and the bounds of each resulting area are computed. The computed bounds are summed up into globalBounds, and UpdateSolver checks whether the winner of this position can be determined from the generalized rules in Section II-D. The solver's internal state is updated with that information. For example, df-pn will update its proof and disproof numbers. This process is repeated until the starting position is solved or resources run out.

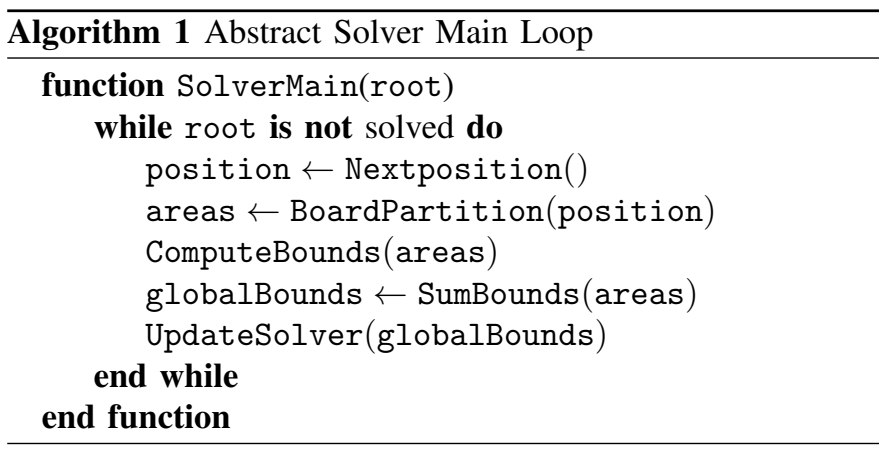

\section{B. Areas in Amazons}

Different detailed analyses of the areas discussed in Section II-A 2 are the main building blocks of the solver. An area in Amazons is a set of 8-connected squares which can contain empty squares and queens of either color. While areas don't contain any burnt-off squares, such squares are often shown in diagrams to improve readability. An area containing only queens, or only empty squares, is called a dead area [11], and is ignored in further analysis since neither player can move there.

In a basic partition, the resulting areas are all independent by construction. In an improved partition, areas can overlap on blockers. Based on whether both players have queens in an area and whether blockers exist, areas can be classified as active, simple territory or blocker territory.

The size of an area is defined as the (width, height) pair of its minimum bounding rectangle.

1) Active Area: An active area contains at least one queen of each color and one empty square. For example, Area $C$ in Figure 3 is a $2 \times 2$ active area. Playing in active area is often good, as it can give a player the chance to block off more squares from the opponent. However, in a zugzwang, playing first is a disadvantage [10].

2) Simple Territory: A simple territory contains queens of only one color and does not overlap with other areas. For example, Area $B$ in Figure 3 is a $1 \times 2$ simple territory for 


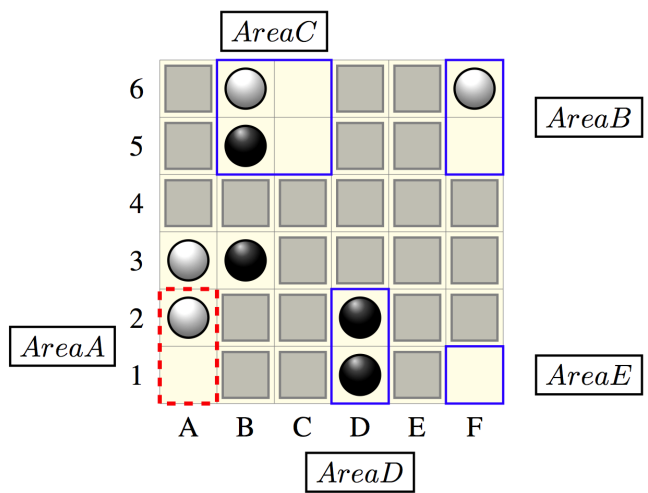

Fig. 3: Area classification example.

White. Since only one player has moves in a simple territory, its absolute value is an integer, the maximum number of moves that the owner can make. Determining this value is not trivial, since territories can be defective and provide fewer moves than the number of empty squares [10]. Examples are shown in Figure 7.

3) Blocker Territory: A blocker territory contains queens of only one color and overlaps with active areas, or with other blocker territories, on its blockers. For example, Area $A$ in Figure 3 is a White blocker territory of size $1 \times 2$. Like simple territories, blocker territories are one-player games with integer value when considered in isolation. However, sometimes a player can profit from moving into an adjacent area and (re-)moving the blockade. Such moves are considered by the solver in its search, but are not used for computing bounds from blocker territories. See the detailed discussion in Section IV-B3.

Having blocker territories separated provides an advantage for both playing and solving the game. From a strategic point of view, blockers provide a player the advantage of securing territory-to-be as well as threatening other parts of the board. For the solver, evaluating the blocker territory separately often yields a stronger bound that leads to a faster proof. Finally, separating out blocker territories shrinks the size and thereby simplifies remaining active areas.

\section{Combinatorial Game Theory Background}

Concepts from combinatorial game theory [12], [13], such as sums of games, canonical form and thermographs, can be used to help evaluate Amazons positions. Each area corresponds to a subgame, and each move affects exactly one subgame. Territories in Amazons correspond to integers in the theory. Many other game values occur in active areas, such as fractions, hot games, and infinitesimals. While the canonical form of an area contains all information needed to play perfectly in a sum of this and other areas, the average complexity of the canonical form grows very rapidly with the size of areas. In contrast, the thermograph of a subgame is of bounded size in practice - fewer than 20 rational numbers suffice to describe all but extremely rare cases.
1) Thermographs: A thermograph - Figure 4(c) shows an example - is a data structure consisting of two sets of line segments, called the left and right scaffold, which describe the minimax value of a game when played with a $\operatorname{tax} t \geq 0$, for Left $=$ Black $=$ positive and Right $=$ White $=$ negative going first, respectively. The scaffolds meet to form a single mast at the temperature of the game. In subzero thermography [14] as used here, the tax can also be negative, down to a value of -1 . This extension of thermography allows stronger comparisons of games by adding detail about a subgame's behavior at low temperatures, which occur frequently on small Amazons boards.

The current work proposes several ways to use thermographs of subgames for computing relaxed bounds on the value of a game, and also by using infinitesimals to improve these bounds and prove that one player can make the last move. While thermographs of two games can not be added to compute the thermograph of the sum, the bounds derived from them can be added, and the information about positive or negative infinitesimal values can be combined, by using the fact that the sum of positive games is again positive, and the sum of negative games is negative. The next subsection formalizes such extended use of bounds.

\section{Generalizing Bounds: Tightened and Relaxed Bounds}

Combinatorial game theory defines a space of game values that is much richer than just integers [13]. Relaxed bounds use two of these concepts, fractions and infinitesimals, as follows:

1) Bounds can be fractions, not just integers. For example, bounds $(G)=\left[-\frac{3}{2}, \frac{5}{16}\right]$ means that $G \geq-\frac{3}{2}$ and $G \leq \frac{5}{16}$ in terms of combinatorial game theory.

2) Both upper and lower bounds can be independently tightened or relaxed.

A tightened bound excludes the number that defines the bound itself, and is written in standard mathematical notation using a round bracket. For example, bounds $(G)=[-3,-1)$ means that the lower bound is $G \geq 3$, while the upper bound is tightened to exclude the value -1 , so $G<-1$. The opposite, a relaxed bound, means that the game value can be infinitesimally outside the bound. A relaxed bound is written by appending the symbols $+\epsilon$ or $-\epsilon$ to the number. For example, the relaxed bound bounds $(G)=[-3,-1+\epsilon]$ means that while $G<-1+\epsilon$ for all $\epsilon>0, G \leq-1$ is not proven. $-1+*$ is an example of a game which would have such a bound. Similarly, bounds $(G)=[-3-\epsilon,-1]$ means that while $G>-3-\epsilon$ for all $\epsilon>0, G \geq-3$ has not been proven. In contrast to simple and tightened bounds, a relaxed bound of 0 cannot be used to establish a win or loss. Both tightened and relaxed bounds can result from using thermographic databases, and more examples of such bounds can be found in Sections II-D1, II-D2 and IV-B2. Addition of bounds uses the following rules, which are applied independently to the upper and lower bounds:

1) If at least one of the bounds is relaxed, then the sum is relaxed. For example, $[1,2+\epsilon]+[1-\epsilon, 3]=[2-\epsilon, 5+\epsilon]$ and $(1,2)+[1-\epsilon, 3+\epsilon]=[2-\epsilon, 5+\epsilon]$. 
2) Otherwise, if at least one bound is tightened, then the sum bound is also tightened. For example, [1,2] + $(1,3]=(2,5]$ and $(1,2)+[1-\epsilon, 3]=[2-\epsilon, 5)$.

3 ) Otherwise, the bound includes the number and is not relaxed. For example, $[1,2]+[1,3]=[2,5]$.

The following rules can determine the winner of a game $G$ from its relaxed bounds $b=$ bounds $(G)$ :

- Black wins if:

$-l(b)>0$

- $l(b)=0$ and the lower bound has been tightened;

- $l(b)=0$, the lower bound is not relaxed, and it is White to move.

- Similarly, White wins if:

- $u(b)<0$;

- $u(b)=0$ and the upper bound has been tightened;

- $u(b)=0$, the upper bound is not relaxed, and it is Black to move.

In all other cases, bounds $b$ do not provide enough information to determine the winner of $G$. However, in Section IV-C2, extra rules are developed which look at some of the components of a sum game in order to recognize more wins.

While the notion of relaxed and tightened bounds is similar to the concept of confusion interval in combinatorial games [13], there are three distinct cases for each bound in the current framework.

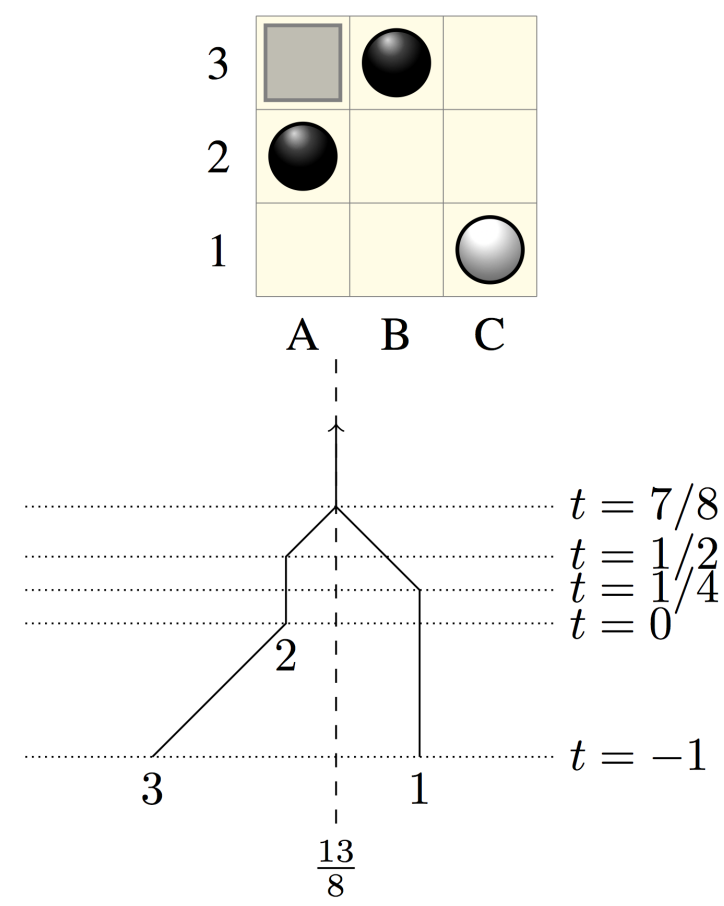

Fig. 4: Active area and its thermograph, with bounds $[1,2+\epsilon]$.

1) Relaxed Bounds from Thermographs for Hot Games: Hot games provide extra moves for the player who makes the first move locally. The left and right stops, the values of the left and right scaffolds at temperature $t=0$, can be used as bounds on the value of a game as follows: If the slope of the scaffold is vertical below $t=0$, then the bound is exact. If it is diagonal, then the game is confused with this value, and the bound must be relaxed.

For example, Figure 4 shows an active area $a$ and its thermograph, with a left stop of 2 and right stop of 1 computed at temperature 0 . The lower bound of 1 is exact since the thermograph is vertical at $t=0$, but the upper bound of 2 is relaxed, since the slope is diagonal there, so bounds $(a)=[1,2+\epsilon]$.
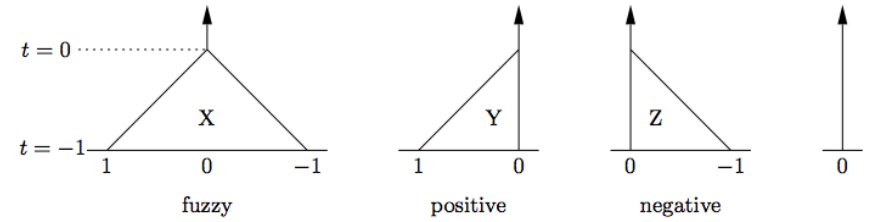

Fig. 5: The four thermographs of loopfree infinitesimals [14, Figure 17].

2) Thermograph Bounds for Infinitesimal Games: In infinitesimal games, no player can gain additional moves, but in close games they can be very important for determining who can make the last move. Infinitesimals can be grouped into four types depending on which player gets to make the last move. In positive games, Black gets the last move no matter who goes first, while in negative games, White does. Games of value 0 are second player wins, while all other infinitesimals are fuzzy, meaning a first-player win locally. An important property of subzero thermography is that the type (but not the exact value except in the case of 0) of an infinitesimal is preserved in its thermograph. Figure 5 shows these graphs.

The corresponding relaxed bounds on an infinitesimal $G$ are $[0,0]$ for $G=0,(0, \epsilon]$ for $G>0,[-\epsilon, 0)$ for $G<0$ and $[-\epsilon,+\epsilon]$ for fuzzy $G$.

The simplest fuzzy infinitesimal is $*=\{0 \mid 0\}$, the game where either player can move first and move exactly once, ending the game. Area $\mathrm{A}$ in Figure 11 is an example. * has the property that $*+*=0$. This is exploited in the solver by recognizing games of value * and dealing with them separately from the rest of the sum, by simply removing pairs of * from the sum. Other fuzzy infinitesimals are also counted separately since a single fuzzy infinitesimal is a first player win. Details on how these facts are used for improved winner detection are given in Section IV-C2.

\section{ENDGAME DATABASES IN AMAZONS}

In board games in general, endgame databases can be either full-board or partial-board depending on what information is stored. A full-board endgame database contains pre-computed exact values of game positions. For a convergent game, where the number of possible full-board states decreases as the game progresses, such databases reduce the depth of the search. Large endgame databases have been successfully used to solve checkers, Nine Men's Morris, Awari and many other games [15]. 
For divergent games such as Amazons or Go, it makes no sense to build such full-board databases. However, if the game board can be split into independent subgames, then partialboard endgame databases can provide perfect information for at least some subgames, which helps the overall solving process. An advantage of partial-board databases is that they can be used regardless of the full board size, as in Tegos' Amazons-playing program Antiope [5].

Amazons endgame databases are built for the three different area types of Section II-B. This section focuses on describing the new blocker territory databases, since the other two types have been built and used before.

\section{A. Building Blocker Territory Databases}

The computational technique used to generate the blocker territory databases is retrograde analysis. This process starts by generating terminal positions whose values are known statically, then computes positions that lead to a terminal position directly by doing a 1-ply lookup. Next, all positions 2 ply away from terminal positions can be solved by a 1-ply lookup, and so on until either some stopping criteria such as the memory limit, or the beginning of the game is reached.

Retrograde analysis for blocker territory databases is complicated by the special behavior of blockers. When a blocker moves into a blocker territory, it must shoot back to its origin square in order to prevent the opponent from getting into this territory. This property is called the blocker constraint. After this initial move, a blocker turns into a normal queen which can move and shoot freely. Therefore the values in a blocker territory database depend on positions where some of the blockers have become normal queens: the positions in a blocker territory database of size $w \times h$ with $B$ blockers, $Q$ normal queens and $E \leq w \times h-B-Q$ empty squares potentially depend on positions of the same size with $b$ $(0 \leq b \leq B)$ blockers, $Q+B-b$ normal queens and $e$ $(0 \leq e \leq E-B+b)$ empty squares. For example, Figure 6 shows that the $3 \times 3$ blocker territory database with 2 blockers, 1 normal queen and 6 empty squares depends on $3 \times 3$ positions with 1 blocker, 2 normal queens and up to 5 empty squares, as well as on $3 \times 3$ positions with 0 blockers, 3 normal queens and up to 4 empty squares. Note that each move by a blocker also burns off a square and thereby reduces the number of empty points left.

$$
3 \times 3, B=2, Q=1, E=6
$$

Fig. 6: Blocker territory database dependency example.

After the databases covering the dependencies are built, retrograde analysis can be applied in the usual way.

\section{B. Databases Used}

1) Simple territory databases: The simple territory databases are similar to those of Tegos [5]. However, the blocker territory database entry format was used for the simple territory databases rather than line segment graphs as in Tegos' implementation. 48 simple territory databases were computed: $1 \times n, 2 \times n$ and $3 \times n$ databases for all $2 \leq n \leq 6$, except for $3 \times 6$ with 4 queens. The database statistics are summarized in [16, Table B.1].

2) Active area databases: Active area databases use the implementation by Enzenberger which is part of the code base for the Amazons program Arrow [17]. Each entry stores the position as in the territory databases and a reference to its corresponding thermograph [16]. The active area databases are built without blocker constraints. Therefore, they cannot be used to query a remaining active area when blocker territories are partitioned out in an improved partition. All nontrivial $1 \times n$ and $2 \times n$ databases were built for $2 \leq n \leq 6$, plus $3 \times n$ for $3 \leq n \leq 5,3 \times 6$ with at most two queens each, and the four smallest $4 \times 4$ databases. [16, Table B.2] shows their statistics.

3) Blocker territory databases: Similarly, 106 blocker territory databases were built and summarized in [16, Table B.3].

\section{IMPROVING AREA AND GLOBAL BOUNDS}

This section discusses how the bounds of different types of areas are computed. Section IV-A shows how bounds of each type of area are initialized by using heuristics, or estimated by local search. Section IV-B describes how tighter bounds can be obtained from databases. Section IV-C describes how local bounds are combined to try to find a winner for a given full board position.

\section{A. Computing Bounds by Heuristics}

1) Simple Territory: Since there is no easy way in general to determine whether a simple territory can be completely filled or not (see Section II-B), as in [11] a simple plodding heuristic is used to quickly compute a basic lower bound $p$. Then the bounds of a simple territory with $v$ empty squares are initialized to $[p, v]$ for Black, and $[-v,-p]$ for White. The heuristic counts the number of moves each queen can make by one sampled sequence of plodding moves, which move to a neighboring empty square and shoot back. Each visited empty square is marked and not used by other queens. For example, the bounds of the position shown on the left of Figure 7 are $[1,2]$ since the heuristic can only make 1 move with this Black queen. In this defective territory, 1 is the true number of moves, but in general the heuristic just returns a lower bound.
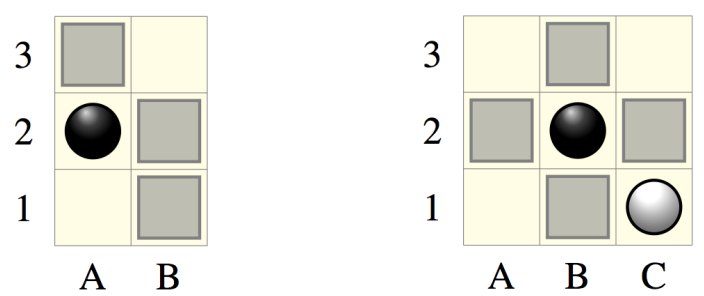

Fig. 7: Territory Bounds: Left: Simple territory, bounds $[1,2]$. Right: Blocker territory, bounds $[1,3]$. 
2) Blocker Territory: The bounds of a blocker territory are initialized in the same way as a simple territory. When filling a blocker territory, the plodding heuristic starts by using normal queens, and uses blockers only when necessary.

For example, the position shown on the right in Figure 7 is a Black blocker territory with blocker $B 2$. The bounds computed by the heuristic are $[1,3]$ since the blocker has to shoot back when it moves, thus blocking its access to the other empty squares.

3) Active Area: The bounds of an active area with $v$ empty squares are initialized to $[-v, v]$, then improved by either static evaluation or local $\alpha \beta$ search. Static evaluation improves bounds by identifying safe moves for the players. This is often only a small improvement, but is fast enough to compute for all active areas. Local search computes bounds for an active area by exhaustive $\alpha \beta$ search. For performance reasons, it is only used for relatively small non-database positions.

4) Safe Moves: The concept of safe moves was introduced in [11] in order to improve bounds on an Amazons area. Safe moves are guaranteed for a player, since the opponent cannot eliminate them. Several examples will be shown below.

5) Static Evaluation: A queen needs at least one adjacent empty square (AES) to make a move. The purpose of static evaluation is to find out if there are safe moves for either player. Bounds are improved according to the following rules:

1) For every Black safe move, the lower bound is increased by 2 ;

2) For every White safe move, the upper bound is decreased by 2 .

Each safe move changes a bound by 2 since one safe move for a player also means one less potential move for the opponent. For example, if Black has two safe moves and White has one safe move in an active area with $v$ empty points, then the improved bounds are $[-v+4, v-2]$.

How many safe moves a queen can make depends on its AES status, the opponent's queen distribution and whose turn it is to move next. Four rules for finding safe moves were defined in [11], and are also implemented in this solver. The current work contributes two new static evaluation rules, Rule 5 and Rule 6, as follows:

Rule 5 takes the origin square of the opponent queen which blocks a single AES into account. For example, in Figure 8 on the left, the White queen $A 2$ has 1 AES $B 2$ which the Black queen $D 2$ can block by $\mathrm{D} 2-\mathrm{C} 3 \times \mathrm{B} 2$ as shown in the figure. However, in blocking this AES, Black has to free the White queen $D 1$, so one safe move can be claimed for the two White queens combined.

Rule 6 is similar to Rule 5 for a queen with 2 AES. In Figure 8 on the right, the White queen $D 1$ has 2 AES and the Black queen $A 3$ can block both by moving to $C 1$ and shooting to $C 2$. However, this opens up $A 3$ for White queen $B 4$, and White can claim one safe move for $D 1$ and $B 4$ combined.

There are potential dependencies amongst safe moves for different queens [11]. Therefore, static evaluation is only applied at most once for each player in an active area.
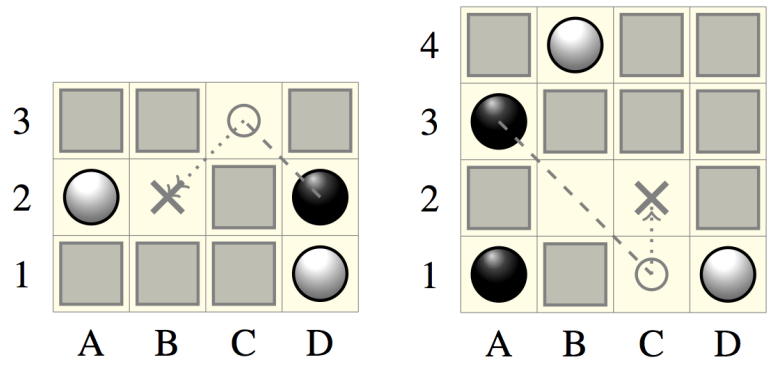

Fig. 8: New static evaluation Rules 5 and 6 for queens with 1 and 2 AES.

6) Local Search: Bounds of an active area can be computed on the fly by local $\alpha \beta$ search. Two such searches are done for each area, one for each player going first. The search score is the difference in number of moves on the board between the first player and the opponent. Passes are allowed for both players even if they have other legal moves, in order to handle zugzwang positions correctly. A search ends after two consecutive passes or when there are no more empty squares.

Let the local search value from the first player's point of view be $v_{w}$ and $v_{b}$ for White and Black moving first in an active area $A$ respectively. Then the bounds of $A$ are set to $\left[v_{w}-\right.$ $\left.\epsilon, v_{b}+\epsilon\right]$. For efficiency, local searches are only performed on non-database positions with total number of (queens plus empty squares) less than 9 .

\section{B. Computing Bounds from Databases}

1) Simple Territory Databases: When a simple territory is encountered during a search, it is queried against the simple territory database with the corresponding size and number of queens. If the query is successful, its exact value $v$ is returned and the bounds for this territory are set to $[v, v]$. As an example, for the defective territory in Figure 7 on the left, the query returns the exact value of 1 and the bounds are set to $[1,1]$.

2) Active Area Databases: An active area encountered during the search is queried against the active area database with the corresponding size and number of queens of each color. If the query succeeds, the thermograph of this area is retrieved and (possibly relaxed) bounds for the area are computed as in Section II-D1.

3) Blocker Territory Databases: Blocker territories are the result of an improved partition of an active area. In the case that the original active area is not in the database as a whole, an improved partition is computed. If no blocker territories can be partitioned out, then the bounds of this active area are computed as in Section IV-A3. If the improved partition succeeds, then the bounds of the resulting blocker territories and active areas are computed separately. A blocker can be used to improve the bounds of at most one area that it is part of. For example, Figure 9 shows a blocker territory delimited by the dashed line, containing one normal queen $A 1$ and one blocker $B 2$. This blocker can either be used to help fill the blocker territory, or to move in the remaining active area. 


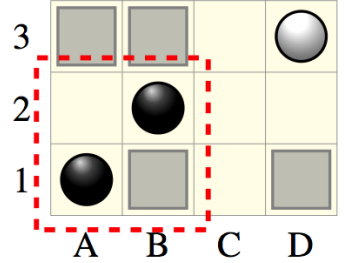

Fig. 9: Blocker territory with 1 normal queen and 1 blocker.

In the current implementation, neither active area databases nor local searches consider the blocker constraint. Bounds on the remaining active areas are only computed with static evaluation as in Section IV-A3, with the additional constraint that blockers that are used in other areas must not be used to find safe moves (either by themselves or combined with other queens). The more blockers can be spared from use in blocker territories, the better the chances for achieving tighter active area bounds. For example, in Figure 9, if the blocker $B 2$ is used for filling the blocker territory, then it cannot be used to improve the bounds of the remaining active area $r$. The bounds of $r$ are therefore [-4,2] since White has a safe move. If queen $A 1$ is used to fill $A 2$, then the blocker $B 2$ can be used to improve the bounds of $r$ to $[-2,2]$ because it has a safe move in $r$.

Unfortunately, in order to reduce the size of blocker territory databases, the current implementation does not store the information about which queens are used for filling a territory. Therefore, a query needs to assume that all blockers and normal queens are used for filling, even if the territory can be filled with only a small subset of them, like queen $A 1$ in the example above.

4) Changing the Query Position: To ameliorate this problem, three heuristics select a subset of all the queens in a blocker territory $B$, to create a new query position $B^{\prime}$ which reduces the number of blockers used. The remaining blockers are not chosen for filling this territory, and are free to be used for other adjacent areas. One drawback of eliminating queens from the query position $B^{\prime}$ of a blocker territory $B$ is that if $B^{\prime}$ is defective, it is not sure whether $B$ is defective or not. In terms of bounds, bounds $(B)=\left[u\left(\right.\right.$ bounds $\left.\left(B^{\prime}\right), e\right]$, where $e$ is the number of empty squares in $B$. If $B^{\prime}$ is non-defective, then $B$ is also shown to be non-defective since it can be filled completely. For example, if the square $C 2$ containing a blocker is removed from the blocker territory in Figure 10, then this smaller territory within the solid line becomes defective, so the computed bounds are $[1,2]$. Details of the queen selection process are described in [16, Chapter 4.2.3].

\section{Combining Bounds}

After a game position $G$ is partitioned into areas, the bounds of each area are computed and added to yield a single global bound. This bound is then checked against the rules in Section II-D to try to determine the winner of $G$. For simple territories computed by heuristics or from the databases, blocker territories computed by heuristics or from

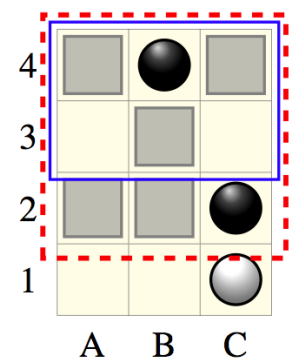

Fig. 10: Territory becomes defective without the blocker $C 2$.

the databases, assuming blockers blocking multiple blocker territories are handled properly as stated in Section IV-B3, and for active areas computed by static evaluation and local search, the combination is just bounds summation. Combining active areas computed from thermographs is more complex since more detailed information about these games in their thermographs can be used to achieve better bounds. The handling of these games is explained in the next two subsections.

1) Handling Hot Game Bounds From Thermographs:

Bounds on a sum game can be improved by simulating a one move lookahead. If at least one hot game is present, the best improvement is achieved by assuming that the next player will move in a hot game with widest bounds $b_{w}$, which are bounds for which the difference $u\left(b_{w}\right)-l\left(b_{w}\right)$ is largest. Then in the sum, $b_{w}$ can be improved as follows: If Black is to play, raise the lower bound $l\left(b_{w}\right)$ (which could also be a relaxed bound of $l\left(b_{w}\right)-\epsilon$ or tightened) to $u\left(b_{w}\right)-\epsilon$. Analogously, if White is to play, lower the upper bound from $u\left(b_{w}\right)$ to $l\left(b_{w}\right)+\epsilon$. For example, if the widest bounds in the sum are $[-5,2]$, they can be replaced by $[2-\epsilon, 2]$ if it is Black's turn, and by $[-5,-5+\epsilon]$ if White plays next.

This replacement yields valid global bounds since it corresponds a conservative estimate of the effect of the first player moving in this subgame and committing to keep replying to opponent's moves here until the stop value is reached. Because of the minimax principle, a bound computed from restricting the first player's strategy is a lower bound (from the player's point of view) on the best result that player can achieve. Using the next player's privilege of moving first in this way precludes using it again for infinitesimals, as in the next section.

2) Handling Infinitesimals: In principle, bounds for infinitesimals can be handled just like other games, according to the addition rules in Section II-D. However, games of value * and other fuzzy infinitesimals are collected separately in the solver, to take advantage of the fact that $*+*=0$ and to identify some more wins as explained below. If the next move privilege was not used on a hot game, it can potentially be used with an infinitesimal to prove more wins. The rules below are for Black, with the rules for White obtained easily by negating all values and outcomes. Let $b$ be the bounds on a sum game excluding infinitesimals, with $l(b)=0$, and the lower bound not relaxed. Then Black as the next player wins if:

- no other infinitesimals exist and there is an odd number of $* \mathrm{~s}$; 
- no other infinitesimals exist and there is a single fuzzy infinitesimal;

- all other infinitesimals are positive and there is either an odd number of $*$, or a single fuzzy infinitesimal, but not both.

In all these cases Black wins by playing first in the fuzzy infinitesimal (which might be a *) and leaving a game $G^{L} \geq 0$ for White.

3) Board Evaluation Examples: A Win by *: Figure 11 shows a $6 \times 6$ Amazons endgame position with 9 empty squares left. The board is partitioned into 4 active areas and all the areas can be looked up in the databases. Area $A$ is recognized as a $*$, and areas $B, C$ and $D$ are all the integer 0 . Therefore, the sum game is evaluated as $0+*$ and the first player, Black in this case, can win by moving in the $*$, leaving 0 for White.

Without databases, area $A$ would still be recognized as a *, but $B, C$ and $D$ would be searched locally, resulting in each of them bounded by $[-\epsilon,+\epsilon]$. The exact nature of these infinitesimals would remain unknown, and the winner could not be determined without search.

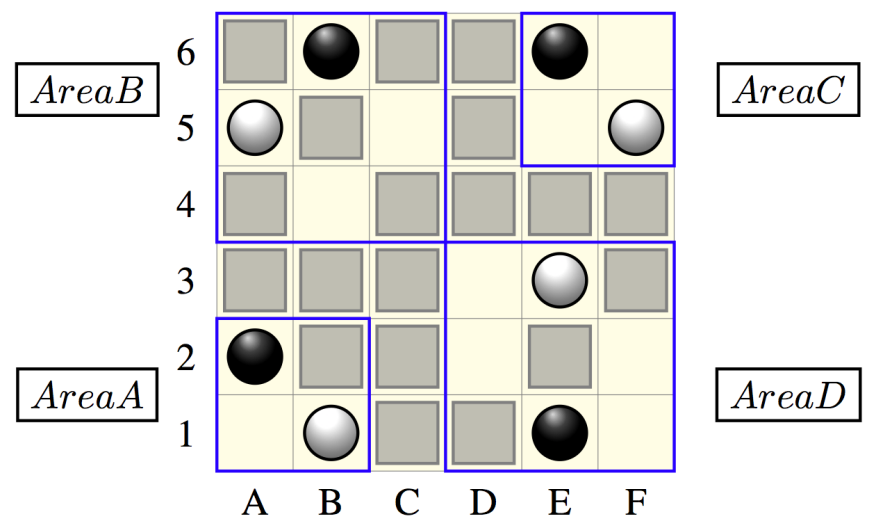

Fig. 11: Black to move can win, value *.

A Local Search Win: Figure 12 shows a $6 \times 6$ Amazons endgame position with 6 empty squares left. Area $B$ is recognized as a $*$. Area $C$ is a simple territory of value 1. Area $A$, of size of $3 \times 6$ with two queens each, is not in the current databases. Local search results in bounds $[-\epsilon, 2+\epsilon]$. The global bounds (both with or without the *) are $[-\epsilon, 2+\epsilon]+1=[1-\epsilon, 3+\epsilon]$, securing a win for Black.

Without local search, Area $A$ has bounds $[-2,2]$ since both players have one safe move there. The global bounds become $[-2,2]+1+*=[-1,3]+*$, and no winner can be determined from these bounds.

\section{EXPERIMENTS AND RESULTS}

\section{A. Solving Test Cases}

Two Amazons solvers were developed within the Arrow framework: $a b$ is a $\alpha \beta$-based solver similar to the one used in [11], and $d f-p n$ is the new df-pn based solver. In the first experiment, the performance of the solvers and databases was compared using the same test data as in [11], which contains positions selected from three test games named $f 1, f 2$ and $f 3$.

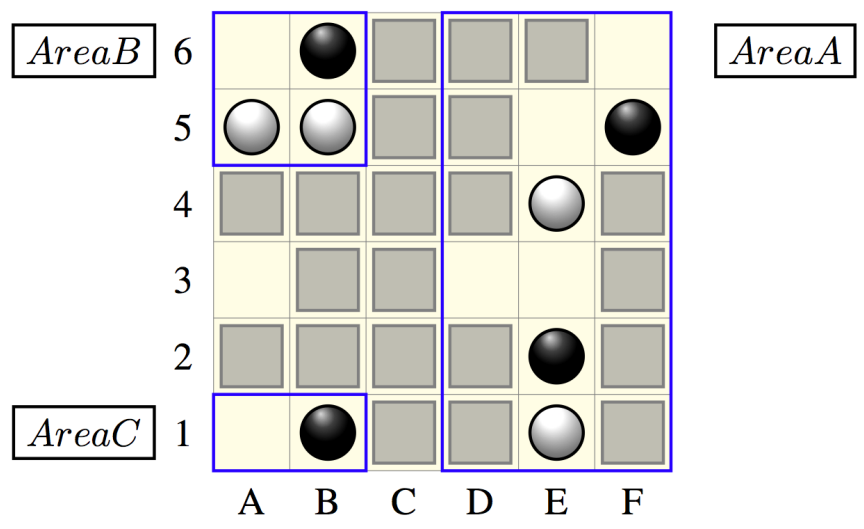

Fig. 12: White to move, global bounds $[1-\epsilon, 3+\epsilon]$.

$f 1$ and $f 2$ are $5 \times 5$ games and $f 3$ was played on $6 \times 6$. The test set is available at [18]. The configuration of the solvers is as follows: $a b$ uses a hash table with $2^{25}$ entries and no databases, and $d f$-pn has a hash table with $2^{22}$ entries. This is roughly fair in terms of memory usage since individual table entries are larger. $d f-p n$ is evaluated in all 8 combinations of including/excluding the three types of databases.

The search results for $f 1, f 2$ and $f 3$ are shown in Figure 13. In the largest test cases, $a b$ exceeded the time limit of 10000 seconds per search. For each graph, the horizontal axis is labelled with the test case number for even-numbered test cases. The number of empty squares in the test cases increases from left to right. The vertical axis shows the number of nodes searched to solve each case on a logarithmic scale. $d f$-pn outperforms $a b$, sometimes by more than an order of magnitude, in all large test cases and most small ones. Using the databases sometimes reduces the number of nodes by another order of magnitude. Most $d f$-pn results are clustered into two groups, with the versions including the combinatorial game databases outperforming the ones without. In contrast, both types of territory databases yield only small improvements. This is due in part to the small size of the board, which does not allow many large territories to be formed. Most very small territories are already evaluated correctly by the non-database static evaluation.

\section{B. Solving $5 \times 6$ Amazons: A First Player Win}

The $d f$-pn solver with a hash table of $2^{30}$ entries plus the databases as specified in Section III-B weakly solved the $5 \times 6$ starting position shown in Figure 1 as a first player win. The first move was fixed as White B1-B4xD4, and all 157 possible replies for Black were refuted, running them as separate search tasks but with a shared hash table on one Quad-Core AMD Opteron(tm) Processor 8384 running at $2.7 \mathrm{GHz}$. Out of 157 moves, 9 were solved instantly by a 1 ply search through a transposition table lookup. For example, after the Black move 2. E5-C5xC4 is refuted by a line starting with White 3. D1$\mathrm{D} 3 \mathrm{xC} 2$, the Black move 2. E5-C5xC2 can be immediately refuted by White 3. D1-D3xC4. Both lines lead to the same position after three moves, swapping who plays the arrow shots 

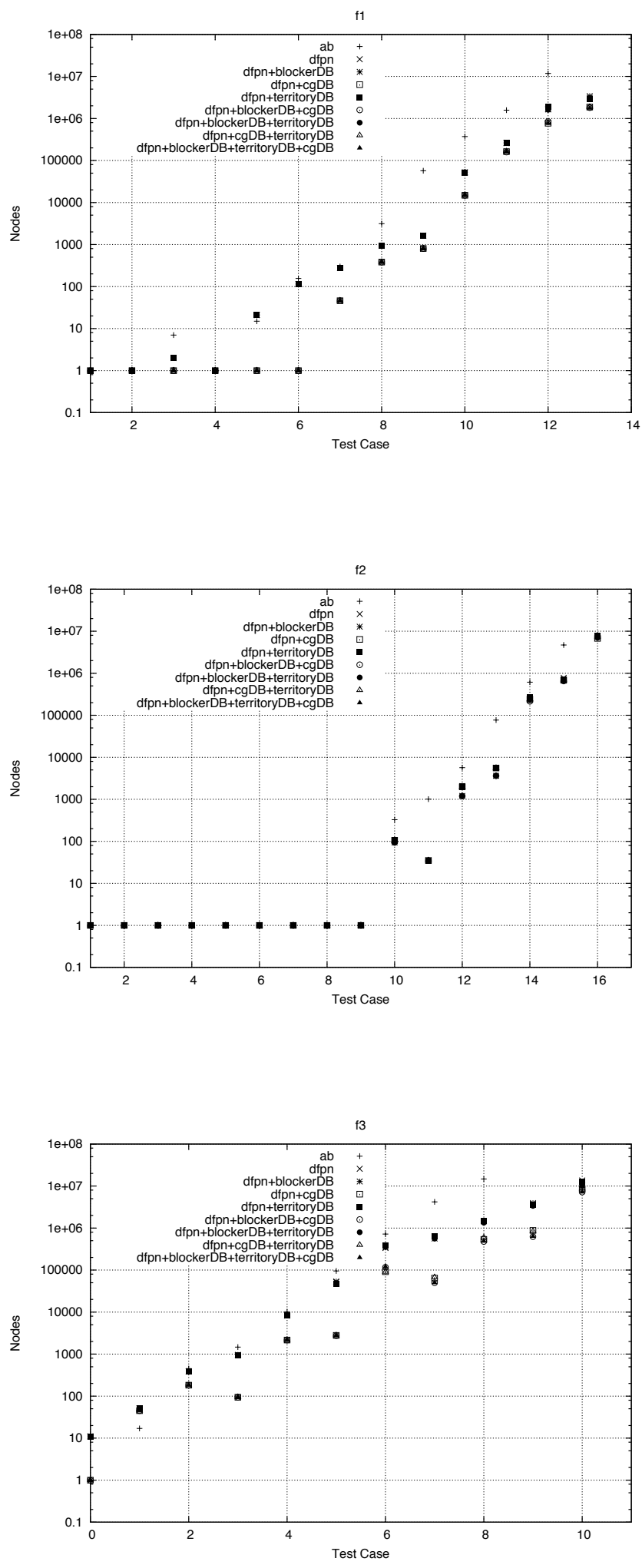

Fig. 13: Search results of $f 1$ (top), $f 2$ (middle), $f 3$ (bottom). to $\mathrm{C} 2$ and $\mathrm{C} 4$. A total of 47 Black moves was refuted in under 1 second each. In contrast, the hardest moves 2. E5E3xE6 and E5-E3xE5 took between three and four hours each to refute. A total of 12 Black moves took over 90 minutes each. A complete strategy for winning $5 \times 6$ Amazons was computed and verified with a proof tree checking program. A typical difficult line in the proof is shown in Figure 14. For the entire line, there is only one active area of size $5 \times 6$. In the final position, shown at the bottom of Figure 14, White has two blocker territories of value -1 and -2 respectively, while Black has one blocker territory of value 2 . The remaining active area in the top left corner is evaluated as 0 since both players can find a safe move. The sum of these subgames is -1 , a White win.

C. Solving Early $6 \times 5$ and $6 \times 6$ Positions

\begin{tabular}{|r|r|r|r|r|}
\hline Move Nr & no DB & DB & df-pn & total games \\
\hline 22 & $\mathbf{7 4}$ & $\mathbf{7 4}$ & $\mathbf{7 4}$ & 74 \\
21 & $\mathbf{8 8}$ & $\mathbf{8 8}$ & $\mathbf{8 8}$ & 88 \\
20 & 82 & 94 & $\mathbf{9 8}$ & 98 \\
19 & 84 & 95 & $\mathbf{1 0 0}$ & 100 \\
18 & 38 & 84 & $\mathbf{1 0 0}$ & 100 \\
17 & 56 & 76 & $\mathbf{1 0 0}$ & 100 \\
16 & 21 & 49 & $\mathbf{1 0 0}$ & 100 \\
15 & 31 & 53 & $\mathbf{1 0 0}$ & 100 \\
14 & 13 & 28 & $\mathbf{1 0 0}$ & 100 \\
13 & 13 & 26 & $\mathbf{1 0 0}$ & 100 \\
12 & 10 & 22 & $\mathbf{1 0 0}$ & 100 \\
11 & 9 & 11 & $\mathbf{1 0 0}$ & 100 \\
10 & 0 & 4 & $\mathbf{1 0 0}$ & 100 \\
9 & 1 & 1 & $\mathbf{1 0 0}$ & 100 \\
8 & 0 & 1 & 96 & 100 \\
7 & 0 & 0 & 91 & 100 \\
6 & 0 & 0 & 53 & 100 \\
5 & 0 & 0 & 36 & 100 \\
\hline
\end{tabular}

TABLE II: Solving $6 \times 5$ positions from 100 games. Columns show number of positions solved statically without databases, statically with databases, and with 500 seconds of df-pn search. Results with all positions solved in bold.

To further evaluate the performance of static evaluation, databases and the solver on harder and larger boards, a set of representative $6 \times 5$ and $6 \times 6$ positions was created. For both board sizes, 100 self-play games were played by Arrow2. These test sets are available on [18]. Starting from the end of each game, positions were tested with the static evaluation without databases, the static evaluation using all databases, and a 500 second $d f$-pn search using all databases and a $2^{22}$ entry hash table.

Tables II and III show the results on $6 \times 5$ and $6 \times 6$ boards respectively. On $6 \times 5$, static evaluation without databases can solve most positions for moves 19-22, a significant fraction of positions from move $15-18$, and a small number of positions for moves 11-14, before dropping to almost zero for earlier positions. With databases, wins are recognized about 2-3 moves earlier, which is a very significant improvement given the exponential growth of the search tree. With search, 91 of 100 games on $6 \times 5$ are solved after 7 moves, then performance drops quickly. 


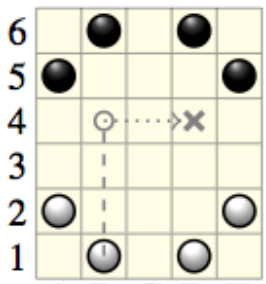

A B C D E

a) $B 1-B 4 \times D 4$

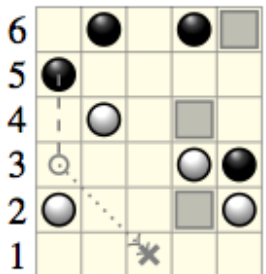

A B C D E

d) $A 5-A 3 \times C 1$

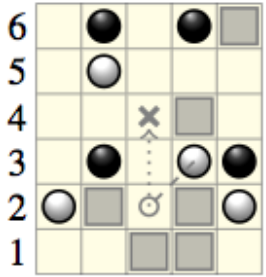

A B C D E

g) $D 3-C 2 \times C 4$

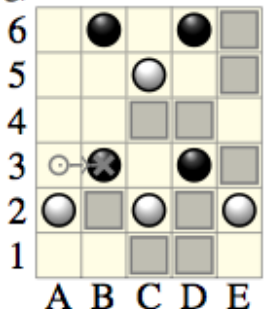

j) $B 3-A 3 \times B 3$

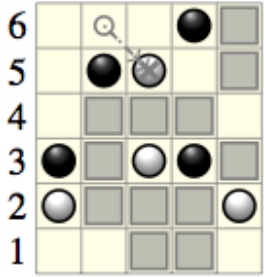

A B C D E

m) $C 5-B 6 \times C 5$

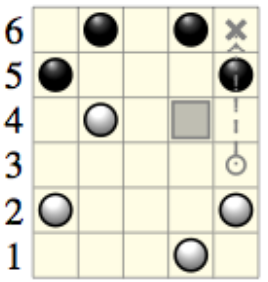

A B C D E

(b) $E 5-E 3 \times E 6$

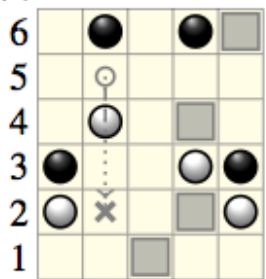

A B C D E

(e) $B 4-B 5 \times B 2$

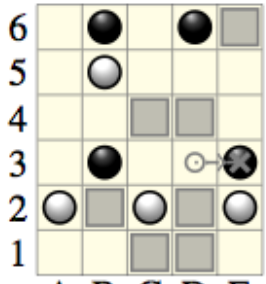

A B C D E

(h) $E 3-D 3 \times E 3$

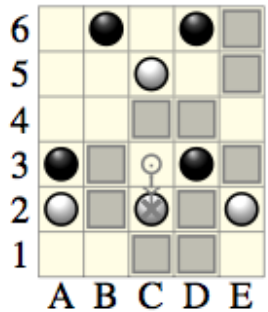

(k) $C 2-C 3 \times C 2$

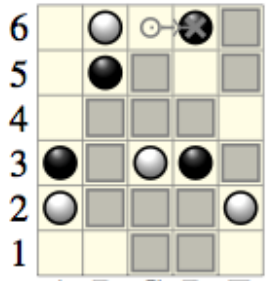

A B C D E

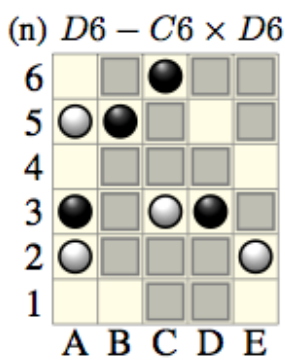

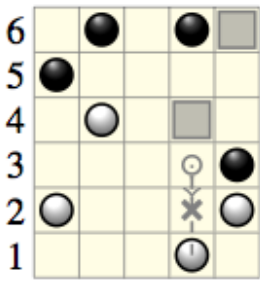

A B C D E

(c) $D 1-D 3 \times D 2$

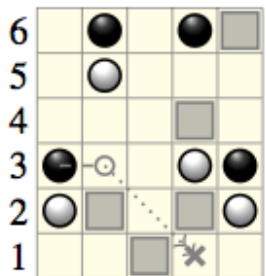

A B C D E

(f) $A 3-B 3 \times D 1$

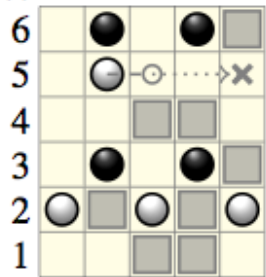

A B C D E

(i) $B 5-C 5 \times E 5$

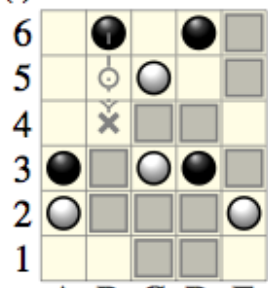

A B C D E

(l) $B 6-B 5 \times B 4$

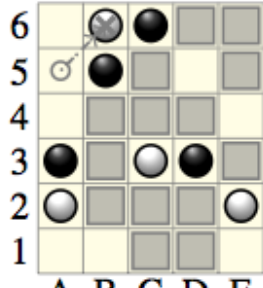

A B C D E

(o) $B 6-A 5 \times B 6$

.

$$
\text { the }
$$
constraint such that the remaining active areas in an improved partition can also be queried, and blocker databases could be augmented to identify the blocking queen(s).

\section{B. Parallel Computing}

The solvers described above are single-threaded. For solving larger problems such as $6 \times 6$, it makes more sense to 

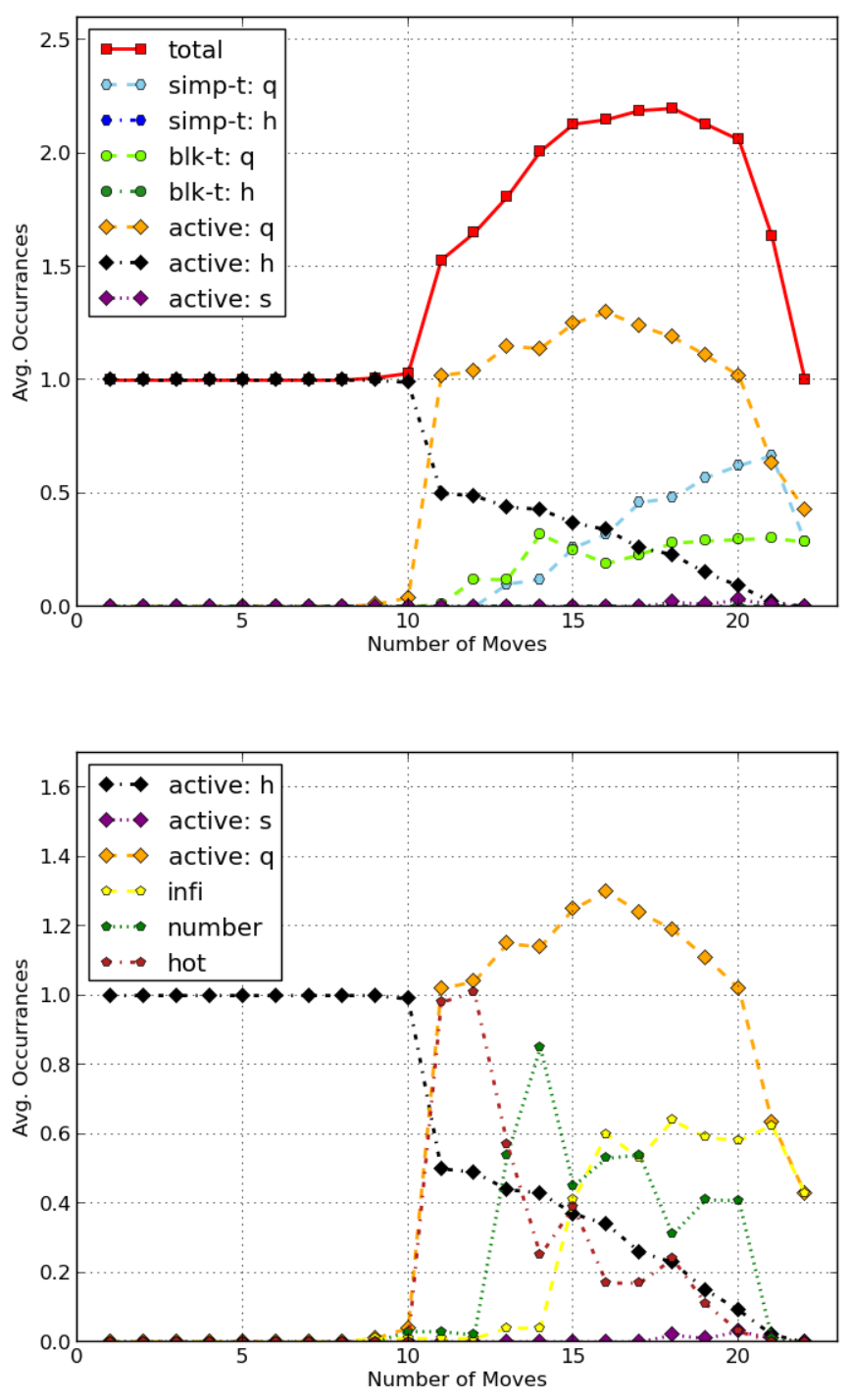

\begin{tabular}{|r|l|}
\hline Legend & \multicolumn{1}{|c|}{ Meaning } \\
\hline total & the sum of all types of areas \\
\hline simp-t: $\mathrm{q}$ & simple territories queried from the databases \\
\hline simp-t: $\mathrm{h}$ & simple territories computed by heuristics \\
\hline blk-t: $\mathrm{q}$ & blocker territories queried from the databases \\
\hline blk-t: $\mathrm{h}$ & blocker territories computed by heuristics \\
\hline active: $\mathrm{q}$ & active areas queried from the databases \\
\hline active: $\mathrm{h}$ & active areas computed by heuristics \\
\hline active: $\mathrm{s}$ & active areas computed by local searches \\
\hline infi & infinitesimals in active: $\mathrm{q}$ \\
\hline number & numbers in active: $\mathrm{q}$ \\
\hline hot & hot games in active: $\mathrm{q}$ \\
\hline
\end{tabular}

Fig. 15: Area frequency on $5 \times 6$ board. Top: all types. Below: active areas, details. have a multi-threaded/distributed solver with a master process/machine generating unsolved nodes and a farm of slave processes/machines actually solving them [16].

\section{Search Improvements}

- Local search improvements. Currently, the local search module does not use the databases and does not consider the blocker constraint. Both techniques should speed up these searches.

- Proof number initialization. The proof and disproof numbers of a newly created node can be initialized to indicate how easy or hard it is expected to solve [19]. This could direct the solver towards easier to prove subtrees.

- Area caching. Caching search results of areas that are not in the databases but arise frequently in the search could give the solver a further performance boost.

\section{ACKNOWLEDGMENTS}

Financial support was provided by NSERC, the Natural Sciences and Engineering Research Council of Canada.

\section{REFERENCES}

[1] J. Kloetzer, H. Iida, and B. Bouzy, "The Monte-Carlo approach in Amazons," in Proceedings of the Computer Games Workshop, 2007, pp. 185-192.

[2] M. Buro, "Simple Amazons endgames and their connection to Hamilton circuits in cubic subgrid graphs," in Lecture Notes in Computer Science, T. Marsland and I. Frank, Eds., vol. 2063. Springer, 2001, pp. 250-261.

[3] T. Furtak, M. Kiyomi, T. Uno, and M. Buro, "Generalized Amazons is PSPACE-complete," in IJCAI2005. Morgan Kaufmann, 2005, pp. 132-137.

[4] E. Berlekamp, "Sums of $N \times 2$ Amazons," in Institute of Mathematics Statistics Lecture Notes, ser. Monograph Series, no. 35, 2000, pp. 1-34.

[5] T. Tegos, "Shooting the last arrow," Master's thesis, University of Alberta, Edmonton, AB, Canada, 2002.

[6] R. Snatzke, "New results of exhaustive search in the game Amazons," Theoretical Computer Science, vol. 313, no. 3, pp. 499 - 509, 2004.

[7] J. Kloetzer, H. Iida, and B. Bouzy, "Playing Amazons endgames," ICGA Journal, vol. 32, no. 3, pp. 140-148, 2009.

[8] — - "A comparative study of solvers in Amazons endgames," in $C I G$, H. P. and L. Barone, Eds. IEEE, 2008, pp. 378-384.

[9] Y. Okada, H. Kuroda, and Y. Kanada, "Subgame database for Amazons," in The 8th Game Programming Workshop in Japan 2003, T. Ito and T. Nakamura, Eds., 2003, pp. 82-89, in Japanese.

[10] M. Müller and T. Tegos, "Experiments in computer Amazons," in More Games of No Chance. Cambridge University Press, 2002, pp. 243-260.

[11] M. Müller, "Solving $5 \times 5$ Amazons," in The 6th Game Programming Workshop (GPW 2001), 2001, pp. 64-71.

[12] J. Conway, On Numbers and Games. Academic Press, 2000.

[13] E. Berlekamp, J. Conway, and R. Guy, Winning Ways for Your Mathematical Plays. Academic Press, 1982.

[14] E. Berlekamp, "The economist's view of combinatorial games," in Games of No Chance: Combinatorial Games at MSRI. Cambridge University Press, 1996, pp. 365-405.

[15] H. van den Herik, J. Uiterwijk, and J. van Rijswijck, "Games solved: now and in the future," Artif. Intell., vol. 134, no. 1-2, pp. 277-311, Jan. 2002.

[16] J. Song, "An Enhanced Solver for The Game of Amazons," Master's thesis, University of Alberta, Edmonton, AB, Canada, 2012. [Online]. Available: http://hdl.handle.net/10402/era.29646

[17] M. Müller, "The Amazons-playing Program Arrow2," http://webdocs.cs. ualberta.ca/ mmueller/amazons/arrow.html, accessed on Nov 14, 2012.

[18] J. Song and M. Müller, "Amazons test games," 2013. [Online]. Available: http://webdocs.cs.ualberta.ca/ mmueller/amazons/games.html

[19] A. Nagai, "Df-pn Algorithm for searching AND/OR trees and its applications," Ph.D. dissertation, Department of Information Science, University of Tokyo, 2002. 